\title{
A Case of Acute Myocardial Injury - MINOCA or Myocarditis?
}

\author{
Camelia Libenciuc', Răzvan-Andrei Licu', Istvan Kovacs,2, Monica Chitu1,2, Imre Benedek1,2 \\ 1 Clinic of Cardiology, Emergency Clinical County Hospital, Târgu Mureș, Romania \\ 2 Center of Advanced Research in Multimodality Cardiac Imaging, CardioMed Medical Center, Târgu Mureș, Romania
}

\section{CORRESPONDENCE \\ Răzvan-Andrei Licu \\ Str. Gheorghe Marinescu nr. 50 \\ 540136 Târgu Mureș, Romania \\ Tel: +40 265212111 \\ E-mail: licu.razvan@yahoo.com}

\section{ARTICLE HISTORY}

Received: July 9, 2020

Accepted: August 25, 2020
Camelia Libenciuc • Str. Gheorghe Marinescu nr. 50, 540136 Târgu Mureș, Romania. Tel: +40 265212 111, E-mail: camelialibenciuc@gmail.com

Istvan Kovacs • B-dul 22 Decembrie $1989 \mathrm{nr} .76$ 540124 Târgu Mureș, Romania. Tel: +40 265217333 , E-mail:kov_istvan@yahoo.com

Monica Chitu • B-dul 22 Decembrie 1989 nr. 76 540124 Târgu Mureș, Romania. Tel: +40 265217333 , E-mail: iulia.chitu@yahoo.com

Imre Benedek • B-dul 22 Decembrie 1989 nr. 76, 540124 Târgu Mureș, Romania. Tel: +40 265217333 , E-mail: imrebenedek@yahoo.com

\begin{abstract}
Myocardial infarction with non-obstructive coronary arteries (MINOCA) has been defined as clinical presentation of an acute coronary syndrome with laboratory evidence of myocardial necrosis, but with coronary stenosis of less than $50 \%$ on coronary angiography. On the other side, myocarditis is an inflammatory response triggered by viral, bacterial, fungal, lymphocytic, eosinophilic, or autoimmune myocardial injury, which may be associated with elevated myocardial necrosis serum biomarkers. We present the case of a young male patient with acute chest pain, ST-segment elevation, and high-sensitivity troponin levels of 22,162 ng/L.
\end{abstract}

Keywords: myocarditis, MINOCA, acute myocardial injury, troponin levels

\section{INTRODUCTION}

Myocardial infarction with non-obstructive coronary arteries (MINOCA) occurs in $6 \%$ of patients with myocardial infarction and has been defined as the clinical picture of an acute myocardial infarction with less than $50 \%$ stenosis in the main epicardial coronary arteries. ${ }^{1}$ The proposed mechanisms involved in MINOCA include coronary spasm, myocarditis, microvascular dysfunction, Takotsubo syndrome, or coronary embolism. Twelve-month mortality rates in patients with MINOCA have been reported up to $4.7 \% .^{2}$ Myocarditis, one of the main differential diagnoses of MINOCA, is an inflammatory response triggered by various viral, bacterial, or fungal infections, but may also be caused by autoimmune disorders. Myocarditis was found in $12 \%$ of young adults, and could lead to dilated cardiomyopathy and subsequent heart failure. ${ }^{3}$ The diagnosis of myocarditis is established on the basis of histological, immunological, immunohistochemical, and molecular criteria; however, the diagnosis and cause of myocarditis are certified by endomyocardial biopsy. Cardiac magnetic resonance imaging (CMR) allows morpho-functional characterization of the myocardium, and it can provide important prognostic information. Furthermore, CMR allows the quantification of myocardial fibrosis and extension of the scar tissue following various disorders that trigger myocardial injury. ${ }^{4}$ 


\section{CASE REPORT}

A 44-year-old male patient with no cardiovascular history presented to the emergency department complaining of acute onset of a constrictive chest pain, irradiating in the left upper limb, lasting more than 20 minutes and accompanied by diaphoresis and dyspnea, which had started 12 hours prior to presentation. Clinical examination revealed a blood pressure of $130 / 85 \mathrm{mmHg}$ and heart rate of 79 beats per minute, and no other signs of organ failure.

The 12-lead ECG performed in the emergency room showed sinus rhythm, intermediate QRS axis, and ST-segment elevation of $0.5-1 \mathrm{~mm}$ in leads DI, DII, aVL, aVF, and V4-V6 (Figure 1).

Blood sampling for detection of myocardial necrosis were obtained and the results revealed an increased highsensitivity troponin I level of 26,162 ng/L, CK-MB of 155.5 $\mathrm{ng} / \mathrm{mL}$, and a total creatine kinase of $1,531 \mathrm{U} / \mathrm{L}$. The FOCUS echocardiography conducted in the emergency room revealed a mildly impaired left ventricular systolic function, with a left ventricular ejection fraction of $50 \%$, with discrete lateral wall hypokinesis, no significant valvular disease, and no modifications in the pericardium. Several criteria of acute posterolateral ST-segment elevation myocardial infarction (STEMI) were identified: chest pain, ST-segment elevation in more than two concordant chest leads, serological evidence of myocardial necrosis, and regional wall motion abnormalities on echocardiographic examination. Therefore, an emergency invasive coronary angiography was performed, which revealed angiographically normal coronary arteries (Figure 2).

During hospitalization, the patient presented dynamic ECG changes consistent with the evolution of an acute myocardial infarction, with the occurrence of negative $\mathrm{T}$ waves in the chest leads which had presented ST-segment elevation (Figure 3). Laboratory tests revealed increased inflammatory serum biomarkers with an erythrocyte sedimentation rate of $69 \mathrm{~mm} / \mathrm{h}$, and a positive C-reactive protein.

Due to the absence of obstructive coronary artery disease upon invasive coronary angiography, a CMR imaging was performed on day 5 of hospitalization, to exclude myocarditis and other non-ischemic myocardial disorders. CMR examination showed the presence of a non-ischemic lesion located in the subepicardial region of the lateral free wall of the left ventricle (Figure 4).

The presence of a non-ischemic lesion on CMR examination, accompanied by elevated inflammatory biomarker levels, leads to a high suspicion of myocarditis. In the absence of an endomyocardial biopsy for a definitive diagnosis, viral serology was performed for detection of various viral infections with possible tropism for the myocardial tissue, including anti-Cytomegalovirus IgM negative antibodies (negative), anti-Cytomegalovirus IgG positive antibodies (positive), anti-Toxoplasma antibodies type IgM (negative) and IgG (positive), anti-rubella antibodies IgM (negative), Ig G (positive), and HIV antibody test

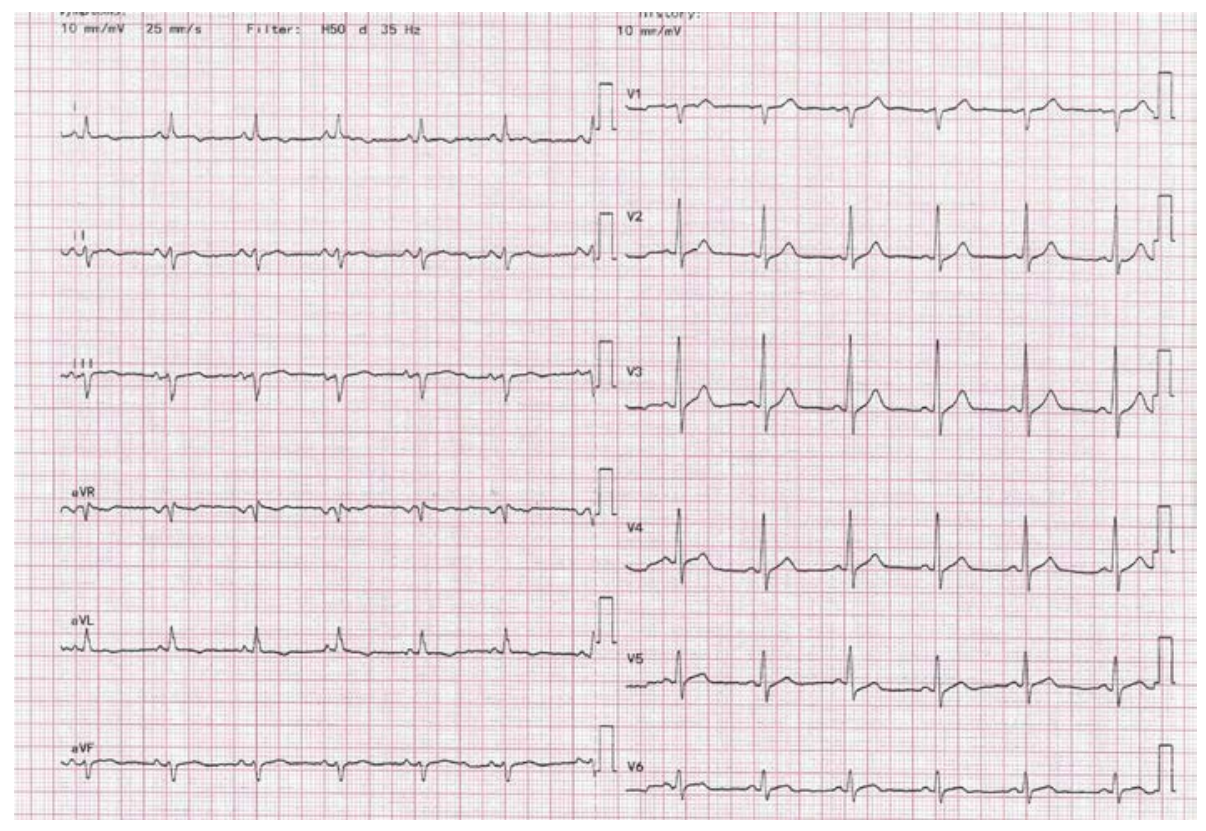

FIGURE 1. ECG performed during chest pain episode showing an ST-segment elevation in DI, DII, aVL, aVF, and V4-V6 leads 

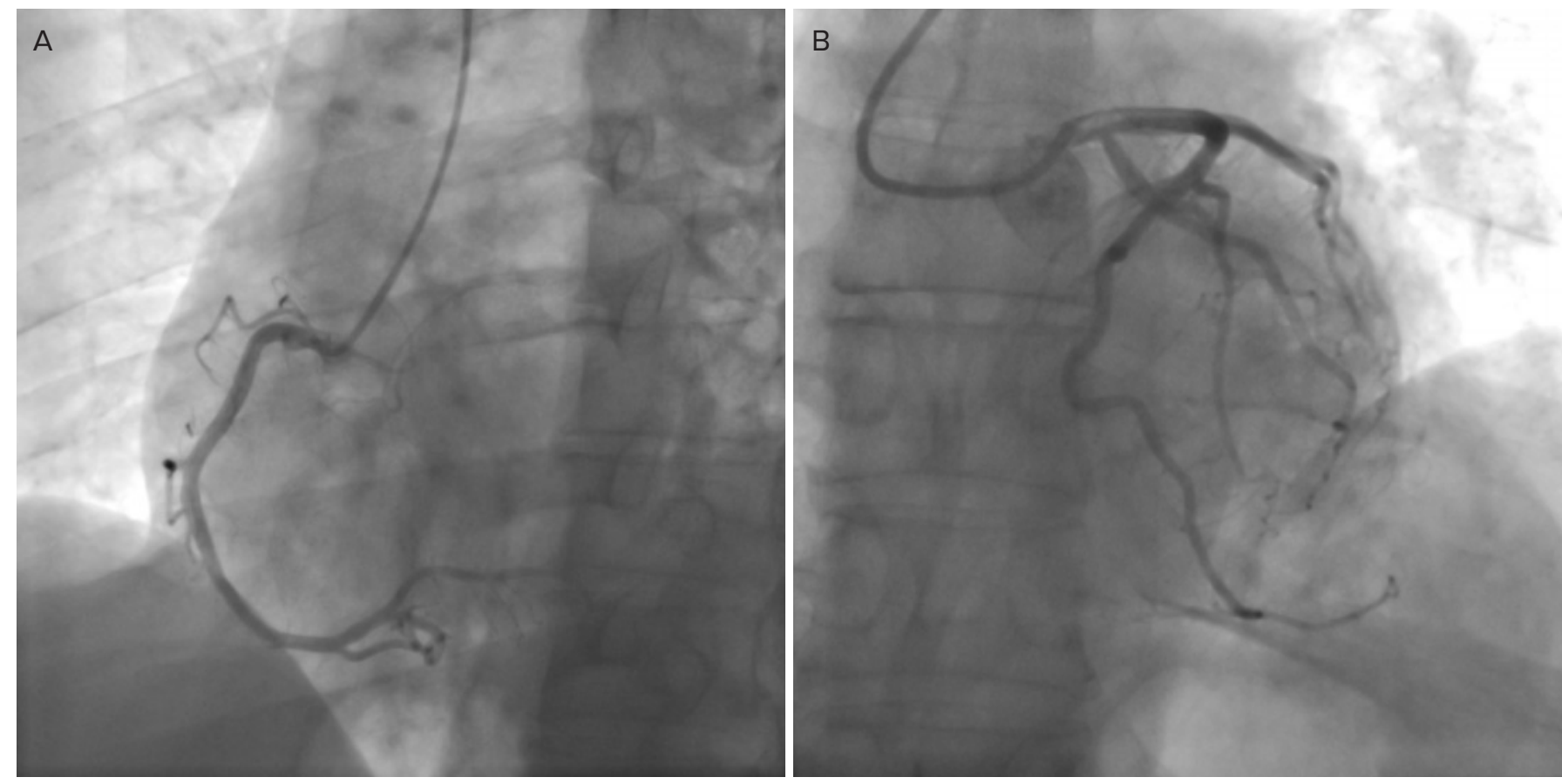

FIGURE 2. Coronary angiography revealing normal epicardial coronary arteries. A - left coronary artery; B - right coronary artery

(negative). The viral serology results were not specific, and a positive diagnosis of viral myocarditis could not be established. However, due to the presence of a non-ischemic lesion on the CMR examination, MINOCA was excluded. The patient was prescribed angiotensin-converting enzyme inhibitors, beta blockers, and a mineralocorticoid antagonist as a triple neurohormonal blockade, in order to prevent left ventricular remodeling following an extensive myocardial injury, albeit non-ischemic. The patient agreed to the publication of his data, and the institution where the patient had been admitted, approved the publication of the case.

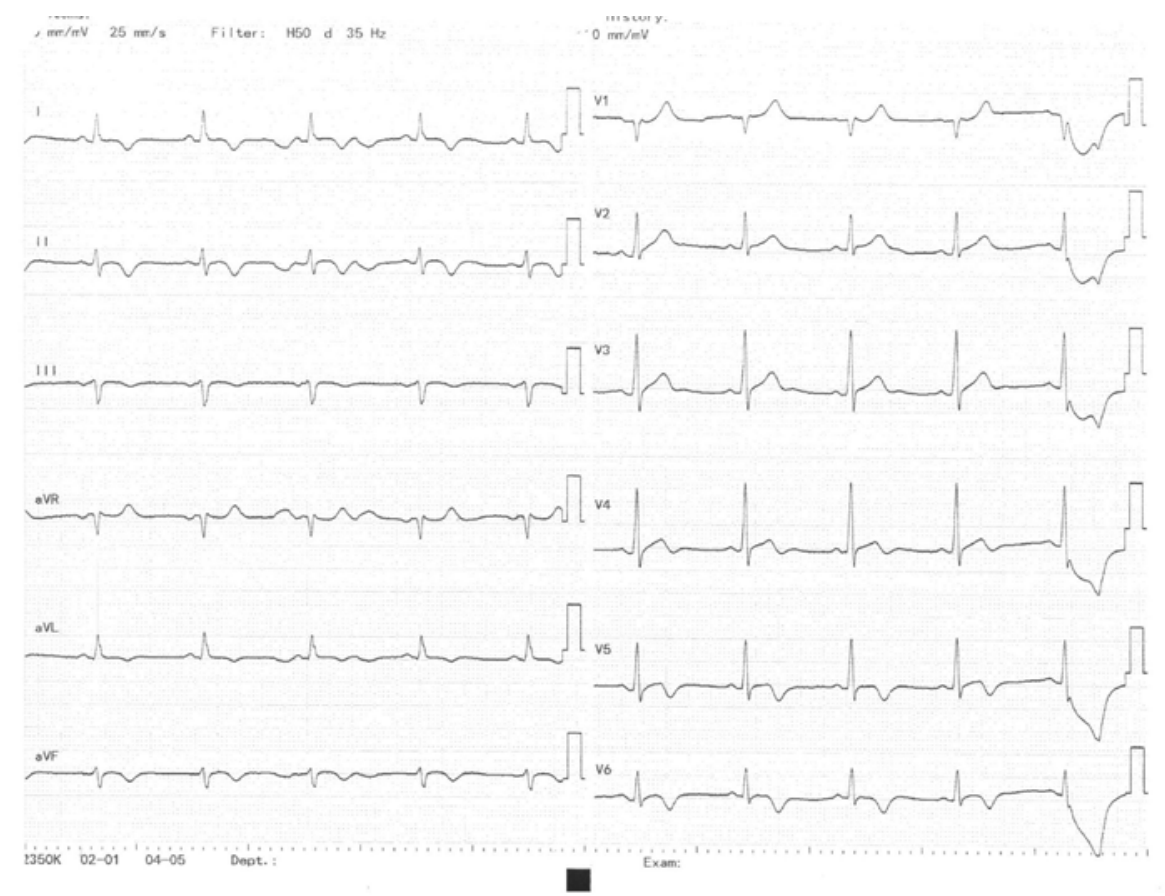

FIGURE 3. ECG on day 3 showed negative T waves in DI, DII, aVL, aVF, and V4 

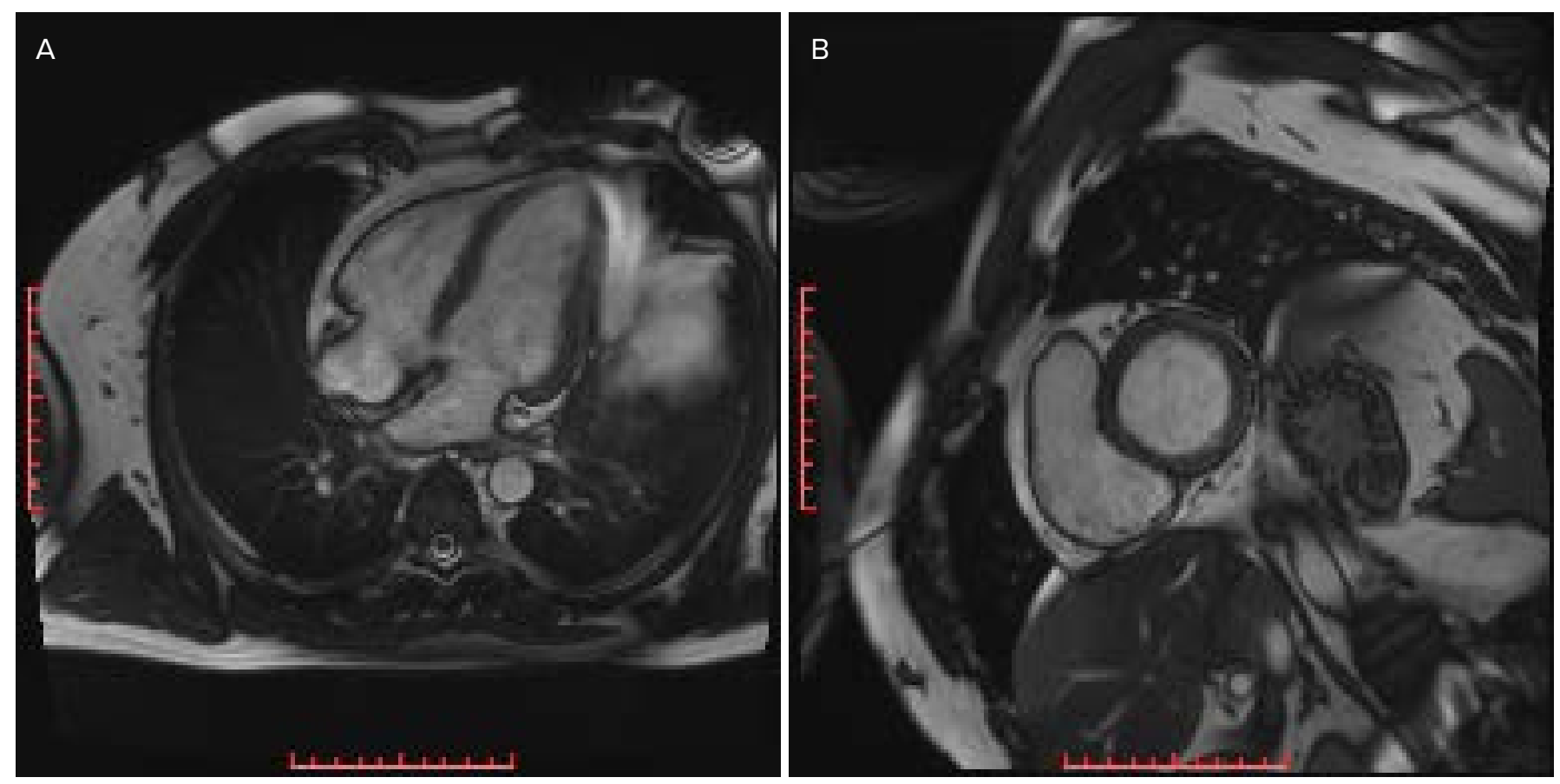

FIGURE 4. Cardiovascular magnetic resonance criteria for myocarditis: regional myocardial edema, hyperemia in images acquired early after contrast injection (A), and inflammatory necrosis in images acquired late ( $>10$ minutes) after contrast injection (B)

\section{DISCUSSIONS}

MINOCA constitutes $6 \%$ to $14 \%$ of cases presenting with acute myocardial infarction. ${ }^{5}$ In the reported case, the patient presented acute chest pain, elevated cardiac necrosis markers, and ST-segment elevation, and was initially diagnosed with acute myocardial infarction. However, emergency coronary angiography demonstrated normal coronary anatomy with no obstructive coronary lesions. This raised an important differential diagnosis problem: MINOCA or myocarditis?

Studies have shown that myocarditis may mimic an acute coronary syndrome at onset, which occurs especially in young males (17-39 years). ${ }^{6}$ Myocarditis shares some clinical and paraclinical characteristics with other diseases, including MINOCA. However, MINOCA does not present severely elevated inflammatory biomarkers or positive serology for viral infections. ${ }^{7}$ Even if in the reported case, the patient had presented immunoglobulin G antibodies for several viral infections with myocardial tissue tropism, including cytomegalovirus, toxoplasma, or rubella, the results were inconclusive and non-specific.

In clinical practice, if a patient presents with signs and symptoms consistent with an acute coronary syndrome, associated with evidence of myocardial necrosis, an emergency invasive coronary angiography is indicated. If the epicardial coronary arteries do not reveal any obstructive lesions, the initial diagnosis of MINOCA is established.
Nevertheless, several differential diagnoses should be excluded. One of the most important differential diagnoses for MINOCA is myocarditis, which is suspected in case of clinical context (chest pain, dyspnea, signs of heart failure), ischemic ECG changes, increased levels of myocardial necrosis enzymes in the presence of non-obstructive coronary arteries, and generally, in patients with a positive history of recent viral infections or autoimmune disorders. ${ }^{8}$ Until recently, endomyocardial biopsy was the most accurate method of diagnosis, but it is rarely used due to its low availability and high rates of complications. CMR imaging remains the most accurate method of diagnosis in acute myocarditis of various causes. ${ }^{9}$ In MINOCA patients, the clinical, laboratory, and angiographic findings may be identical, but the CMR examination presents typical modifications specific for each etiology. ${ }^{10}$ The CMR aspect of myocarditis includes the presence of regional myocardial edema, fibrosis, hyperemia in images acquired early after contrast injection, and inflammatory necrosis in late enhancement. These are generally located in mid- and subepicardial regions of the ventricular wall, whereas the ischemic changes are located in the subendocardial layer of the ventricular wall. ${ }^{11}$

Factors advocating for the diagnosis of myocarditis include young age, male sex, and high levels of C-reactive protein. ${ }^{11}$ MINOCA is an important subtype of acute myocardial infarction, in which, unlike myocarditis, there is no 
acute inflammatory syndrome or positive viral markers in laboratory tests. ${ }^{12}$ In a study conducted in Sweden, it was found that the prevalence of MINOCA in all patients with myocardial infarction recorded over a period of 3 months was $7 \%$, and it was diagnosed in one third of all women with acute coronary syndrome. These results were in high contrast with the prevalence of acute myocarditis that occurred more frequently in young males, accompanied by signs of inflammation, such as fever, and elevated levels of C-reactive protein. ${ }^{13}$ MINOCA patients may also present without ST elevation on the surface ECG. Recent studies show that the presence of ST elevation is directly correlated with early mortality and with the occurrence of longterm complications. CMR imaging helps to differentiate the ischemic from non-ischemic causes that produced the acute coronary event. ${ }^{14}$

The prognosis of acute myocarditis depends on the etiology and severity of symptoms at presentation, especially if it presents as an acute myocardial infarction. In some patients, the clinical evolution may include certain types of arrhythmias or left ventricular dysfunction, which may occur at months or even years after the acute episode, due to myocardial scarring. ${ }^{15}$ A study revealed that $81 \%$ of patients diagnosed with myocarditis presented a complete functional recovery of the left ventricle, while others showed signs of dilated cardiomyopathy after the acute event. ${ }^{16}$ In our case, the patient presented a preserved systolic function, with a left ventricular ejection fraction of $50 \%$, without any other arrhythmic or hemodynamic complications. In terms of treatment, myocarditis can resolve spontaneously in mild cases. To prevent arrhythmias and heart failure, beta blockers, angiotensin-converting enzyme inhibitors, diuretics, and anticoagulants are prescribed. ${ }^{17}$ Etiological treatment is the best therapeutic option, in association with the recommended heart failure therapy. The effectiveness of the medical therapies can be monitored with the evaluation of cardiac troponins, $\mathrm{N}$-terminal pro-B-type natriuretic peptide, and C-reactive protein. Although inflammatory syndrome in myocarditis can resolve spontaneously in mild forms, a well-targeted treatment is often needed. ${ }^{18}$ Despite this, the use of nonsteroidal anti-inflammatory drugs is not recommended because it exacerbates heart failure and increases mortality. ${ }^{19}$ On the other hand, the diagnosis and treatment of MINOCA is similar to that of an acute myocardial infarction with obstructive coronary artery disease, and with the treatment of heart failure if required. Nevertheless, the specific imaging changes associated with the presence of acute inflammatory syndrome and viral markers play a crucial role in differentiating MINOCA from myocarditis. ${ }^{20}$ In our case, the patient showed a good clinical evolution, with remission of cardiac symptoms, and was discharged without any hemodynamic or arrhythmic complications.

\section{CONCLUSIONS}

In most cases of patients with acute coronary syndromes presenting with angiographically normal coronary arteries, the etiological diagnosis remains unclear. The presented case illustrates a young patient with acute myocarditis, with clinical signs and symptoms indicative of an acute coronary syndrome. One of the main differential diagnoses in patients presenting with MINOCA is acute myocarditis. Diagnostic modalities include not only invasive coronary angiography for exclusion of obstructive coronary artery disease, but also CMR imaging. CMR is recommended for all patients presenting with MINOCA, in order to exclude myocarditis or other infiltrative myocardial disorders that may present as acute coronary syndromes in the emergency department.

\section{CONFLICT OF INTEREST}

Nothing to declare.

\section{ACKNOWLEDGEMENT}

This research was supported via the research grant no. 103544/2016, contract number 26/01.09.2016, entitled "Increasing the research capacity in the field of vulnerable plaque imaging, based on advanced nanoparticles, fusion imaging and computational simulation - PlaqueImage", financed by the Romanian Ministry of European Funds, the Romanian Government and the European Union.

\section{REFERENCES}

1. Vogiatzis I, Koutsambasopoulos K, Samaras A, Bostanitis I. Acute Coronary Syndrome With Normal Coronary Arteries: a Case of Spontaneous Spasm Lysis. Med Arch. 2018;72:154-156

2. Pasupathy S, Air T, Dreyer RP, Tavella R, Beltrame JF. Systematic review of patients presenting with suspected myocardial infarction and nonobstructive coronary arteries. Circulation. 2015;13:861-870.

3. Tao Z, Wei M, Shixuan W, et al. Acute myocarditis mimicking ST-elevation myocardial infarction: A case report and review of the literature. Exp Ther Med. 2015;10:459-464

4. Peretto G, Caforioe P, Sala S. Acute myocardial injury, MINOCA, or myocarditis? Improving characterization of coronavirus-associated myocardial involvement. Eur Heart J. 2020; 41:2124-2125.

5. Safdar B, Spatz S, Dreyer P, et al. Presentation, Clinical Profile, and Prognosis of Young Patients With Myocardial Infarction With Nonobstructive Coronary Arteries (MINOCA): Results From the VIRGO Study. J Am Heart Assoc. 2018;7:e009174

6. Costantini M, Tritto C, Licci E, et al. Myocarditis with ST-Elevation Myocardial Infarction presentation in young man. A case series of 11 patients. Int J Cardiol. 2005;101:157-158. 
7. Costantini M, Oreto G, Albanese A, et al. Presumptive myocarditis with ST-Elevation myocardial infarction presentation in young males as a new syndrome. Clinical significance and long term follow up. Cardiovasc Ultrasound. 2011;9:1.

8. Gambetti S, Fucà G, Bressan S, Righi R, Sassone P. Focal acute myocarditis mimicking ST-elevation myocardial infarction: a case report and literature review. G Ital Cardiol (Rome). 2014;15:634-637.

9. Larina O, Mershina E, Sinitsyn V, Andreev D. Cardiac magnetic resonance imaging in the diagnosis of acute myocarditis: a clinical case and a review of literature. Vestn Rentgenol Radiol. 2014;5:54-59.

10. Tornvall P, Gerbaud E, Behaghel A, et al. Myocarditis or "true" infarction by cardiac magnetic resonance in patients with a clinical diagnosis of myocardial infarction without obstructive coronary disease: A metaanalysis of individual patient data. Atherosclerosis. 2015;241:87-91.

11. Tornvall P, Brolin EB, Caidahl K, et al. The value of a new cardiac magnetic resonance imaging protocol in Myocardial Infarction with Non-obstructive Coronary Arteries (MINOCA) - a case-control study using historical controls from a previous study with similar inclusion criteria. BMC Cardiovasc Disord. 2017;17:199.

12. Collste $\mathrm{O}$, Sörensson P, Frick M, et al. Myocardial infarction with norma coronary arteries is common and associated with normal findings on cardiovascular magnetic resonance imaging: results from the Stockholm Myocardial Infarction with Normal Coronaries study. J Intern Med. 2013;273:189-196.
13. Agewall S, Daniel M, Eurenius L, et al. Risk factors for myocardial infarction with normal coronary arteries and myocarditis compared with myocardia infarction with coronary artery stenosis. Angiology. 2012;63:500-503.

14. Hausvater A, Pasupathy S, Tornvall P, et al. ST-segment elevation and cardiac magnetic resonance imaging findings in myocardial infarction with non-obstructive coronary arteries. Int J Cardiol. 2019;287:128-131.

15. Peters A, Loffler A, Kramer C, Salerno M, Kwon Y. Recurrent Myocarditis Imitating ST Segment Elevation Myocardial Infarction. J Med Cases. 2018;9:239-242.

16. Sarda L, Colin P, Boccara F, et al. Myocarditis in patients with clinical presentation of myocardial infarction and normal coronary angiograms. $J$ Am Coll Cardiol. 2001;37:786-792.

17. Magnani J, Dec G. Myocarditis: current trends in diagnosis and treatment. Circulation. 2006;113:876-890.

18. Pankuweit S, Maisch B. Etiology, diagnosis, management, and treatment of myocarditis. Position paper from the ESC Working Group on Myocardial and Pericardial Diseases. Herz. 2013;38:855-861.

19. Pustjens T, Appelman $Y$, Damman P, et al. Guidelines for the management of myocardial infarction/injury with non-obstructive coronary arteries (MINOCA): a position paper from the Dutch ACS working group. Neth Heart J. 2020;28:116-130

20. Tahir S, Nikolaos T. Myocarditis in 2020. Advancements in Imaging and Clinical Management. JACC: Case Reports. 2020. [Epub ahead of print] 